\title{
One Method to Derivate Coulomb's Law between Two Charges
}

\author{
Hongwei Zhang1, Shimeng Feng ${ }^{2 *}$ \\ ${ }^{1}$ Shanghai Qibaodwight High School, Shanghai, China \\ ${ }^{2}$ School of Physics and Astronomy, Shanghai Jiao Tong University, Shanghai, China \\ Email: *smfeng@sjtu.edu.cn
}

How to cite this paper: Zhang, H.W. and Feng, S.M. (2020) One Method to Derivate Coulomb's Law between Two Charges. Journal of Applied Mathematics and Physics, 8, 2880-2885.

https://doi.org/10.4236/jamp.2020.812213

Received: November 15, 2020

Accepted: December 14, 2020

Published: December 17, 2020

Copyright () 2020 by author(s) and Scientific Research Publishing Inc. This work is licensed under the Creative Commons Attribution International License (CC BY 4.0).

http://creativecommons.org/licenses/by/4.0/

(c) (i) Open Access

\begin{abstract}
In electromagnetics, Coulomb's law is a very classic formula. Almost all textbooks give this formula, but none of them give a detailed corresponding theoretical derivation. In order for beginners of physics to better understand the physical meaning of this formula, we explored the source, the physical model and mechanism of this formula. Based on the principle that the interaction between two different fields can generate energy density, which is equal to the pressure, we analyzed the distribution of the electric field energy density as well as the corresponding pressure on the charged surface. Through the rigorous mathematical derivation, we give the theoretical derivation of this formula.
\end{abstract}

\section{Keywords}

Electrostatic Force, Energy Density, Pressure, Coulomb's Law

\section{Introduction}

Coulomb's law is a mathematical description of the electric force between charged objects. According to Coulomb, the electric force for charges at rest has the following properties: If two electrically charged bodies are placed nearby each other there will be an attraction or a repulsion force acting on them depending upon the nature of the charge of the bodies. The formula for the force acting between two electrically charged bodies was first developed by Charles-Augustinde in 1785 . The electric force is proportional to the product of the charge of the charged bodies and inversely proportional to the square of the distance between the center of the charged bodies.

Coulomb's law is summarized from a large number of experiments. Then, almost all the classic electronic books have directly given the formula of Cou- 
lomb's law, and hardly given the theoretical derivation of the formula [1] [2]. Some papers were reported to discuss the formula of the Coulomb force [3] [4] [5], but they did not give out the physical model of the formula, and did not give a detailed mathematical derivation of this formula. For example, Huang Xiulan proved the similarities between the formula of universal gravitation and the formula of Coulomb force [6]; however, he still did not explain how this formula was proved. So far, none of the papers reported the derivation process of this formula. In this paper, we try to do work for the detailed derivation of this formula. Based on the model of point charge, we analyzed the interaction between the electric fields generated by two charges, which produce the energy density at the different position. Using the relationship between energy density and pressure, we derived this formula, which is very meaningful for a deep understanding of the physical nature of electrostatic gravity.

\section{Theory Derivation}

As we all know, there is an electric field around an electric charge, which is a characteristic of electric charge. This electric field has energy and mass. Here we give a classical model of electric field generated by a point charge for the following discussion, as shown in Figure 1. The electrostatic field is described as

$$
\boldsymbol{E}=\frac{q}{4 \pi \varepsilon_{0} r^{3}} \boldsymbol{r}
$$

Here $\boldsymbol{E}$ is the electrostatic field intensity, $\varepsilon_{0}$ is the dielectric constant in vacuum. $q$ is the electric quantity of a charge. $\boldsymbol{r}$ is the position vector from a point to the charge.

For two point charges with a certain distance, the distribution of electric field is shown in Figure 2. The energy density of the electric field should be described as

$$
G=\frac{1}{2} \boldsymbol{D} \cdot \boldsymbol{E}=\frac{1}{2}\left(\boldsymbol{D}_{1}+\boldsymbol{D}_{2}\right) \cdot\left(\boldsymbol{E}_{1}+\boldsymbol{E}_{2}\right)
$$

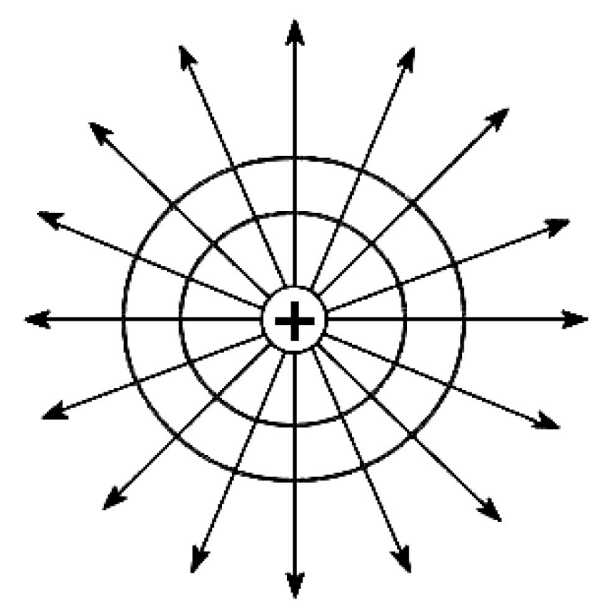

Figure 1. Electric field distribution of a point charge in space. 


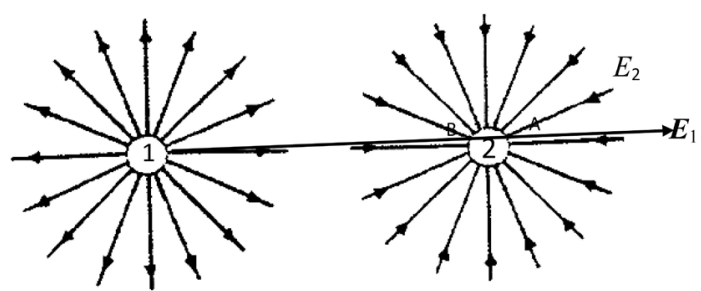

Figure 2. The model of two charges in space. Here 1 is the first charges, 2 is the second charge. $E_{1}$ is the electronic field strength generated by the first charge.

Here $G$ is defined as the energy density, $\boldsymbol{E}_{1}, \boldsymbol{E}_{2}$ is the electrostatic field intensity generated by the first-point charge and the second point charge, respectively (seeing Figure 2), $\boldsymbol{D}_{1}, \boldsymbol{D}_{2}$ is the corresponding electric displacement vector. We expand the Formula (2) to get

$$
G=\frac{1}{2} \boldsymbol{D}_{1} \cdot \boldsymbol{E}_{1}+\frac{1}{2} \boldsymbol{D}_{1} \cdot \boldsymbol{E}_{2}+\frac{1}{2} \boldsymbol{D}_{2} \cdot \boldsymbol{E}_{1}+\frac{1}{2} \boldsymbol{D}_{2} \cdot \boldsymbol{E}_{2}
$$

Using $\boldsymbol{D}=\varepsilon_{0} \boldsymbol{E}$, Equation (2) can be changed to

$$
G=\frac{1}{2} \varepsilon_{0} E_{1}^{2}+\varepsilon_{0} \boldsymbol{E}_{1} \cdot \boldsymbol{E}_{2}+\frac{1}{2} \varepsilon_{0} E_{2}^{2}
$$

Now, we are more interested in the second term of the Formula (4), which represents the energy density generated by the interaction of $\boldsymbol{E}_{1}$ with $\boldsymbol{E}_{2}$. Equation (4) can be written as

$$
G=\frac{1}{2} \varepsilon_{0} E_{1}^{2}+\varepsilon_{0} E_{1} E_{2} \cos \theta+\frac{1}{2} \varepsilon_{0} E_{2}^{2}
$$

Here $\theta$ is the angle between $\boldsymbol{E}_{1}$ and $\boldsymbol{E}_{2}$. The classical physics tells that the energy density is equal to the pressure difference. So, Equation (5) can be written as

$$
P=\frac{1}{2} \varepsilon_{0} B_{1}^{2}+\varepsilon_{0} E_{1} E_{2} \cos \theta+\frac{1}{2} \varepsilon_{0} E_{2}^{2}
$$

Here $P$ is defined as the pressure.

Because the size of the charge is too small, the distance between two point charges is much greater than the radius of charge. Thus, the electric field generated by the first charge around the second charge can be regarded as a uniform electric field. For the following research, we enlarge the size of the second charge, as shown in Figure 3. From Figure 3, it can be clearly observed that the interaction energy of $\boldsymbol{E}_{1}$ and $\boldsymbol{E}_{2}$ is different at the different position of the second charge surface. For example, the corresponding pressure at $A$ and $B$-point of the second-charge is $\left(P_{A}, P_{B}\right)$, respectively,

$$
\left\{\begin{array}{l}
P_{A}=G_{A}=\frac{1}{2} \varepsilon_{0} E_{1}^{2}+\varepsilon_{0} E_{1} E_{2} \cos \theta_{1}+\frac{1}{2} \varepsilon_{0} E_{2}^{2} \\
P_{B}=G_{B}=\frac{1}{2} \varepsilon_{0} E_{1}^{2}+\varepsilon_{0} E_{1} E_{2} \cos \theta_{2}+\frac{1}{2} \varepsilon_{0} E_{2}^{2}
\end{array}\right.
$$

Here $\theta_{1}$ and $\theta_{2}$ are the angle between $\boldsymbol{E}_{1}$ vector and $\boldsymbol{E}_{2}$ vector at the $A$ and $B$-point of the second charge, respectively. 


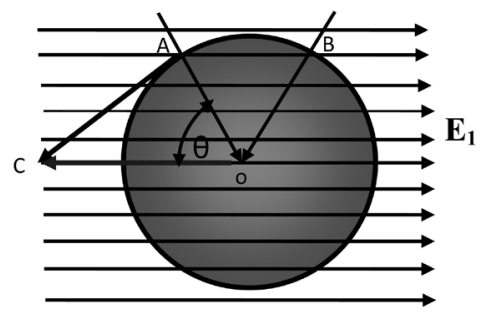

Figure 3. An enlarged view of the electric field force generated on the second charge surface.

The first and third terms in Equation (7) indicate that the corresponding energy density is constant at the different position of the second charge surface, which suggests that the corresponding pressure of the two terms is unchanged on this surface. Thus, the total force generated from them on the whole surface is zero. Only the second term in Equation (7) shows that the corresponding energy density is different at the different positions of the second charge surface, which can produce a pressure difference. That is, the total force generated from the second terms in Equation (7) is not zero, which is the source of the electrostatic force. Therefore, we only consider the calculation of the second term in Equation (7). Here, we set

$$
P_{A}=\varepsilon_{0} E_{1} E_{2} \cos \theta
$$

For the charge surface, the force can be written as

$$
\mathrm{d} \boldsymbol{F}_{A o}=P_{A} \mathrm{~d} S=\left(\varepsilon_{0} E_{1} E_{2} \cos \theta\right)(R \mathrm{~d} \theta)(2 \pi R \sin \theta) \boldsymbol{e}_{A O}
$$

Here $R$ is the radio of the second charge, $\boldsymbol{e}_{A o}$ is the unit vector from $A$-point to $O$-point along the radius of chare (seeing Figure 3 ).

Seeing from Equation (8), there is a difference in energy density at the tangent direction of the charged surface, which also leads to the force through the pressure difference at the direction (seeing Figure 3), which is defined as $\mathrm{d} \boldsymbol{F}_{A C}$ (seeing Figure 3). According to the vector superposition principle of force, we have

$$
\mathrm{d} \boldsymbol{F}_{O C}=\mathrm{d} \boldsymbol{F}_{A O}+\mathrm{d} \boldsymbol{F}_{A C}
$$

Figure 3 shows that $\mathrm{d} \boldsymbol{F}_{A O} \perp \mathrm{d} \boldsymbol{F}_{A C}$. Since the direction of the total force vector (defined as $\mathrm{d} \boldsymbol{F}_{O C}$ ) added up by the above two forces is parallel to the direction of $\boldsymbol{O C}$, which is parallel to the direction of the line between the two charges. Then, $\mathrm{d} \boldsymbol{F}_{O C}$ must satisfy the following relationship,

$$
\mathrm{d} \boldsymbol{F}_{O C}=\frac{\mathrm{d} \boldsymbol{F}_{A O}}{\cos \theta}=\frac{1}{\cos \theta}\left[\frac{q_{1}}{4 \pi \varepsilon_{0} r_{1}^{2}} \frac{q_{2}}{4 \pi \varepsilon_{0} R^{2}} \cos \theta \varepsilon_{0} 2 \pi R \sin \theta R \mathrm{~d} \theta\right]
$$

Equation (11) can be simplified to be

$$
\mathrm{d} \boldsymbol{F}_{O C}=\frac{q_{1} q_{2}}{4 \pi \varepsilon_{0} r_{1}^{2}} \frac{1}{2} \sin \theta \mathrm{d} \theta
$$

Now, through the integration of the whole surface of the charge, we get the formula of the electrostatic attraction force 


$$
\boldsymbol{F}_{O C}=\int_{0}^{\pi} \frac{q_{1} q_{2}}{4 \pi \varepsilon_{0} r_{1}^{2}} \frac{1}{2} \sin \theta \mathrm{d} \theta=\frac{q_{1} q_{2}}{4 \pi \varepsilon_{0} r_{1}^{2}}
$$

Here $\boldsymbol{F}_{O C}$ is the force acting between two charges. Equation (13) is the complete expression of Coulomb's law. This above derivation method proves that the force acting between two objects in space essentially comes from the interaction of electronic fields. The above derivation process also suggests that all forces in space are derived from the difference in energy density produced by the interaction of one field with other fields. There are different fields in the universe, such as electric field, magnetic field, gravitational field, etc. Their interaction will produce energy density. For a certain object, the difference in energy density around it will cause a pressure difference, which can produce a force.

For any formula in physics, there should be many different ways to derive it. Many textbooks only give some conclusive formulas, and did not provide the physical and mathematical explanations of these formulas, which is not conducive to the understanding of students and teachers, and it is not conducive to improving students' interest in physics. The derivation process in this paper can reveal the corresponding physical principles of the formula, which is very meaningful for physicists to deeply understand the physical model and mechanism corresponding to the formula.

\section{Conclusion}

The interaction of the different electric field results in a different energy density difference on the surface of the charge, which causes attraction or repulsion between different charges. Using the difference of energy density, we set a model to derive the formula of Coulomb's law. So far, this derivation method is not reported in other papers. Physics is a discipline that explains the origin of this world and the laws of matter movement. It aims to tell people the truth of this objective world. Therefore, our in-depth thinking on all physical laws can further unlock the truth of this world, which is the value of this paper.

\section{Conflicts of Interest}

The authors declare no conflicts of interest regarding the publication of this paper.

\section{References}

[1] Zhang, Y.M. (1999) Electromagnetism. China University of Science and Technology Press, Hefei, 14-15.

[2] Wu, X.L. (2009) College Physics (2). High Education Press, Beijing, 119-120.

[3] Liu, T.T. and Sun, H.B. (2003) Historical Review of the Establishment of Coulomb's Law. Physics and Engineering, 13, 57-59.(In Chinese)

[4] Wang, F. (2013) The Position of Photon Rest Mass in Coulomb's Law. Journal of Gansu Lianhe University (Natural Science Edition), No. 12, 44-46. (In Chinese)

[5] Lan, Z.G. and Wan, X.L. (2008) The Functions of Analogy Reasoning in the For- 
mulation of Coulomb's Law-A Concurrent Discussion about the Limitations of Analogy Reasoning. Physics Experimentation, No. 11, 37-41. (In Chinese)

[6] Huang, X.L. (1994) The Analogy Reasoning between the Law of Gravitation and Coulomb's Law. Journal of Yunnan Normal University (Natural Science Edition), 14, 49-53. (In Chinese) 